\title{
Comparative study of approaches to bathymetry detection in Nasser/Nubia Lake using multispectral SPOT-6 satellite imagery
}

\author{
Hassan Mohamed ${ }^{1}$, Abdelazim Negm ${ }^{1}$, Kazuo Nadaoka ${ }^{2}$, \\ Tarek Abdelaziz ${ }^{3}$ and Mohamed Elsahabi ${ }^{4}$ \\ ${ }^{1}$ Department of Environmental Engineering, Egypt-Japan University of Science and Technology, Egypt \\ ${ }^{2}$ Department of Mechanical and Environmental Informatics, Tokyo Institute of Technology, Japan \\ ${ }^{3}$ Nile Research Institute, Ministry of Water Resources and Irrigation, Egypt \\ ${ }^{4}$ Department of Civil Engineering, Aswan University, Egypt
}

\begin{abstract}
:
Collecting and analysing bathymetric information is essential for lake management. This is particularly true regarding Lake Nasser/Nubia in Egypt, where accumulated sediment in the lake must be examined. This is typically accomplished through field measurements, which are time consuming and costly. However, remotely sensed imagery provides wide coverage, low cost, and time-saving solutions for bathymetric measurements, especially in shallow areas with high erosion or sediment accumulation, such as at the entrance of Lake Nasser/Nubia. In this study, bagging (Bag) and least square boosting (LSB) fitting algorithms that use reflectance of green and red band logarithms, green/red band logarithms ratio, and blue/red band logarithms ratio are proposed for bathymetry detection. For validation, the proposed approaches were compared with the ratio method (RM) and neural network $(\mathrm{NN})$ conventional methods. Bathymetric data obtained from all methods using SPOT-6 imagery were evaluated by means of global positioning system (GPS) and echo sounder data field measurements. The Bag ensemble outperformed all methods with 0.85 m RMSE, whereas RM, LSB, and NN yielded 1.03, 0.99, and $0.97 \mathrm{~m}$ respectively. The results showed that the proposed approaches outperform and are more accurate than RM conventional method and the Bag approach is more accurate than the NN model when applied over shallow water depths of up to $6.5 \mathrm{~m}$.
\end{abstract}

KEYWORDS bagging; bathymetry; Lake Nasser/Nubia; SPOT images

\section{INTRODUCTION}

Bathymetric estimation of shallow water areas is considered the most important factor in coastal applications, coastal management, and shipping activities (Leu and Chang, 2005). These areas are primarily coastal and lake areas, where sediment movements as a result of tidal changes, wave movements, and floods have caused severe changes at the sea or lake bottom (Lyzenga, 1978, 1985). Therefore, detailed bathymetric information is now required, particularly for these applications.

The conventional methods for detecting bathymetry are single multibeam echo-sounders or airborne LiDAR. Multibeam echo-sounders are considered the most accurate

Correspondence to: Hassan Mohamed, Egypt-Japan University of Science and Technology (EJUST), Postal code 21934, Alexandria, Egypt. E-mail: Hassan.mohamed@ejust.edu.eg method, able to measure bathymetry at up to $8 \mathrm{~cm}$ vertical accuracy in $200 \mathrm{~m}$ water depth. Some equipment can detect the sea floor in water depths of up to $500 \mathrm{~m}$ with adequate vertical resolution. By contrast, Airborne LiDAR is considered suitable for shallow areas and can achieve vertical accuracy of $20 \mathrm{~cm}$ in up to $30 \mathrm{~m}$ water depth (Su et al., 2008). However, these methods are limited by their spatial coverage, extensive costs, and time consumption.

Remote sensing represents a feasible alternative solution for bathymetric detection. Wide coverage, continuous monitoring, and cheaper costs are some of the advantages of remote sensing in bathymetric applications (SánchezCarnero et al., 2014).. Bathymetric estimation using satellite images began in 1970 and the first satellite images used were from Landsat (Lyzenga, 1981). Higher resolution satellites were then launched and used to measure water depth, for instance IKONOS (Stumpf et al., 2003), SPOT-4 (SánchezCarnero et al., 2014), QUICKBIRD (Linda et al., 2011), and WORLD VIEW-2 (Doxani et al., 2012). In these previous studies that examined bathymetry detection, the maximum depth of water in which the seafloor can be detected was $30 \mathrm{~m}$ under specific conditions. In addition, the average mean error was between 10 and $30 \%$ based on water clarity, bottom type, and atmospheric conditions (McIntyre et al., 2006).

Several algorithms have been developed for determining bathymetry according to the relationship between image reflectance values and water depths. Lyzenga (1978) developed the first algorithm based on this linear relationship. This method removes the atmospheric and water surface effect from images wherein the reflected values represent only the water depth. Limitations of this method include the assumptions that the water bottom is homogenous and water clarity is basically the same across the imaged area (Mehdi et al., 2013). Lyzenga (1985) tried to overcome these limitations by using a combination of several imagery bands based on the multiple log linear regression model. Stumpf et al. (2003) developed an algorithm based on the ratio between bands and correlated these values with known water depths. Unfortunately, this latter method has no physical foundation and requires specific parameters to be defined by the user (Sánchez-Carnero et al., 2014).

NNs (Neural Networks) represent a suitable alternative algorithm for bathymetric detection. Ozçelik and Arısoy

Received 1 December, 2015

Accepted 23 February, 2016

Published online 25 March, 2016 
(2010) began using NNs for bathymetric detection because they overcome drawbacks of conventional approaches and, in fact, possess many advantages. For example, field data requirements are reduced and NNs use raw reflectance values regardless of the bottom type or water column factors. Finally, they are more practical and faster than conventional methods. NNs have also been applied in other studies using different satellite images. For instance, Sheela et al. (2013) used IRS P6-LISS III images and Linda et al. (2011) used QUICKBIRD images. The limitation of their usage of neural networks for detecting bathymetry was that they used all image bands as input for the NNs algorithm. In addition, sun glint and certain atmospheric conditions may affect estimation of water depths.

The Bag (Bagging) and LSB (Least Square Boosting) algorithms proposed in this study use green, red, blue/red, and green/red bands logarithms since, after many trials, they were found to provide higher correlation with actual water depth than the other bands. First, images were corrected for atmospheric and sun glint errors. The proposed algorithms were then applied using SPOT-6 imagery over shallow and highly turbid water. All results were evaluated and compared to field measurements of water depth over the same area using an echo-sounder.

\section{STUDY AREA AND DATA}

The study area is the entrance zone of Lake Nasser/Nubia. This area extends to the Sudanese part of Lake Nubia, covering $5 \mathrm{~km}$ in an east-west direction and $5 \mathrm{~km}$ in a northsouth direction (Figure 1). Most of the study area consists of non-uniform, shallow, and highly turbid water with depths up to $6.5 \mathrm{~m}$. It also has a high rate of sediment movement and annual flood changes. Almost the entire lake bottom is covered with clay.

\section{Imagery data}

A SPOT-6 satellite image with four multispectral bands with wavelengths of Blue $(0.455 \mu \mathrm{m}-0.525 \mu \mathrm{m})$, Green $(0.530 \mu \mathrm{m}-0.590 \mu \mathrm{m})$, Red $(0.625 \mu \mathrm{m}-0.695 \mu \mathrm{m})$, and Near-Infrared $(0.760 \mu \mathrm{m}-0.890 \mu \mathrm{m})$ was used to detect bathymetry in the study area. For radiometric corrections, all required parameters were available in an image metadata file. The image had $1.5 \mathrm{~m}$ spatial resolution and was acquired in moderate wave conditions on the 12th of January 2014 which is the same date water depths were measured in the field (see Figure 1).

\section{Echo-sounder data}

Field observations of water depth used for evaluating and calibrating algorithms were acquired by an ODOM hydrographic system echo-sounder instrument (Echotrac model DF 3200 MKII with built-in Differential GPS). The echo-sounder vertical accuracy is $0.01 \mathrm{~m} \pm 0.1 \%$ of depth in water depths ranging from 0.2 to $200 \mathrm{~m}$. About 13,000 water depth points were collected and referenced to the MSL (Mediterranean Sea Level, see Figure 2).

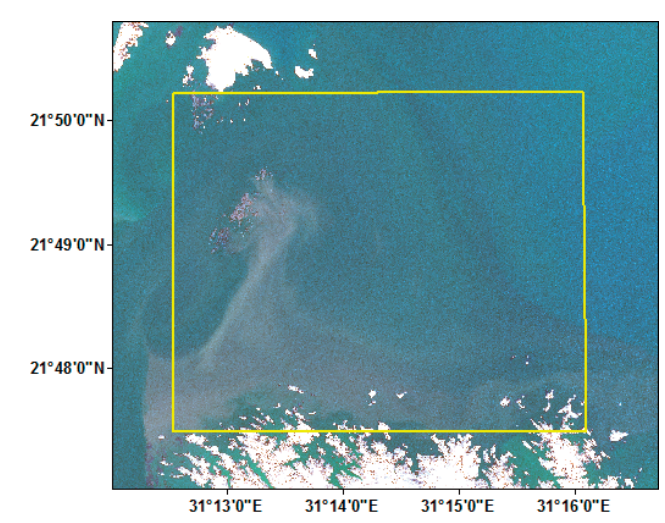

Figure 1. The study area (yellow square) in the entrance zone of Lake Nasser/Nubia, Nile-River-Sudan as a SPOT-6 image

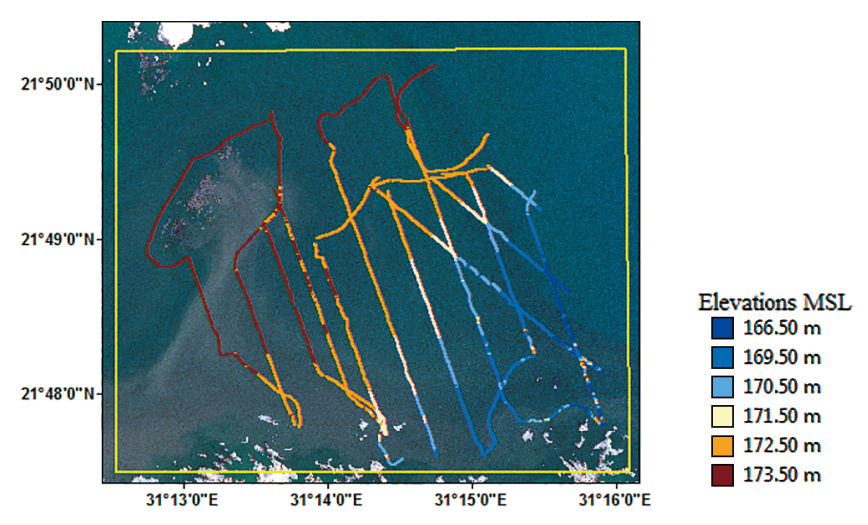

Figure 2. Field bathymetry reference points from echosounder over the study area

\section{METHODOLOGY}

Bathymetric data was calculated from satellite images through three successive steps. First, we converted the digital numbers of image pixels to reflectance values. Second, we corrected the imagery for atmospheric errors. Finally, we corrected the imagery from sun glint errors. The resulting image can be correlated to water depths using field calibrating points. These steps are described in more detail in the following subsections:

\section{Pre-processing of satellite imagery}

We computed the reflectance of each pixel value using the parameters in the metadata file based on the following equation.

$$
\rho \lambda=(\mathrm{Mp} \mathrm{DN}+\mathrm{Ap}) / \sin \theta_{S E}
$$

where, $\rho \lambda$ denotes reflectance of the top of atmosphere reflectance, $\mathrm{DN}$ represents the digital numbers recorded by the sensor, $\mathrm{Mp}$ is the band-specific multiplicative rescaling factor for reflectance, Ap denotes the band specific additive rescaling factor for reflectance, and $\theta_{S E}$ is the local sun elevation angle in degrees. The Mp, Ap, and $\theta_{S E}$ values were available in the image metadata file (.MTL file).

We corrected the reflectance values for atmospheric effects using dark pixel subtraction theory. In this method no 
atmospheric parameters are needed and it is based on the hypothesis that the pixel with the darkest value has no reflection and the remaining value of this pixel came from the atmospheric effect. As a result, an atmospheric correction can be calculated by subtracting all pixels from this pixel using the following equation (Doxani et al., 2012):

$$
\mathrm{Rac}=\mathrm{Ri}-\mathrm{Rdp}
$$

where Rac represents the corrected pixel reflectance value, $\mathrm{Ri}$ is the initial pixel reflectance value $(\rho \lambda)$, and Rdp denotes the darkest pixel value.

We corrected the reflectance values for sun glint errors using the relation between the near-infrared band and other bands (Hedley et al., 2005) based on the following equation:

$$
\mathrm{Ri}^{\prime}=\mathrm{Ri} * \text { bi (RNIR }- \text { MinNIR) }
$$

where $\mathrm{Ri}^{\prime}$ denotes the de-glinted pixel reflectance value, $\mathrm{Ri}$ represents the initial pixel reflectance value, bi is the regression line slope resulting from the correlation between a sample of a visible band reflectance values and NIR band reflectance values over the glinting area, RNIR denotes the corresponding pixel value in NIR band, and MinNIR represents the minimum NIR value in the same sample of the glinting area.

\section{Proposed approaches for bathymetry detection}

Ratio method

The ratio method depends on the difference in attenuation degree between bands; one band may be greater or less than the others in terms of the degree of attenuation. Therefore, this phenomenon can be used to correlate the ratio between bands to the difference in water depth (Stumpf et al., 2003). This approach overcomes the problem of sea bottom variability, especially regarding sea grass or microalgae areas (Su et al., 2008) because the change in sea bottom albedo affects both bands similarly. However, a change in water depth has a greater effect on the bands which are attenuated highly (Michael, 2009). Stumpf et al. (2003) prove the uniqueness of their approach to the log linear inversion model. The following equation describes the ratio model $(\mathrm{Su}$ et al., 2008).

$$
Z=m_{1}\left(\ln \left(n \lambda_{1}\right)\right) /\left(\ln \left(n \lambda_{2}\right)\right)-m_{o}
$$

where $m_{o}, m_{1}$, and $n$ are constant coefficients, and $\lambda_{1}$ and $\lambda_{2}$ are two bands with corrected reflectance values. The constant coefficients can be determined through correlating field points with the pixel values of the same points and thereby used to detect bathymetry over the whole study area.

NN method

The supervised approach of the multi-layer perception (MLP) model with the back propagation (BP) method as a training algorithm can be used to demonstrate the non-linear relationship between input and output data (Rumelhart et al., 1986). This approach consists of three parts. The input layers act as neurons, which, in this case, are the multispectral image band values. The hidden layer is used to determine the network training process. Finally, the output layer represents the water depths in this case (Mehdi et al., 2013). The BP algorithm tries to reach a pre-defined level of accuracy. Therefore, it starts with initial weights to find the values with the least number of errors by comparing network outputs with actual values in an iterative process (Behzad, 2014).
The transference of net inputs to the hidden layer occurs by means of the log sigmoid function. In addition, the linear function is used to transfer from the hidden layer to node outputs (Ozçelik and Arısoy, 2010). Because it is highly recommended in training middle-sized MLP NNs, the Levenberg-Marquard training algorithm was used to train the BP for weight and bias values updating (Ananth, 2004).

\section{LSB fitting algorithm}

The basic purpose of boosting is to increase the strength of weak learners by combining them to produce a more powerful model (Hastie et al., 2008). In boosting theory, multiple models are developed in sequence and higher weights are assigned to learners which are difficult to fit in regression problems (Quinlan, 2006). Initial weak learners learn sequentially and simple models are fitted to data, then all data is analysed for error prediction. Data samples that are difficult to fit are then identified. The next step is to create models which attempt to focus on these difficult data samples by assigning them higher weights and identifying the correct prediction (Mochizuki and Murakami, 2012). Therefore, boosting is a technique to improve the performance of a primary model by converting a series of weak learners into robust predictors (Ihler, 2012).

\section{Bag ensemble fitting algorithm}

Breiman (1996) suggested Bag as an ensemble learning algorithm to improve prediction model performance, regression, and classification accuracy. His goal was to overcome overfitting problems and reduce algorithm variance. The main objective of bagging theory is to create independent samples with replacements from the training set, and then generate a fitting model to each bootstrap sample. Finally, all generated models are aggregated by averaging in regression problems (Shivali and Vishakha, 2014). This process can be particularly useful for improving the results of unstable algorithms as regression trees and NNs. The results are always more favorable than when using a single model (Inoue and Kilian, 2005). The Gini diversity index can be used to split each node in order to assign a criterion for impurity or error. Splitting is finished when the Gini index reaches zero and the results are pure split nodes (Ozlem and Oguz, 2012).

\section{RESULTS AND DISCUSSION}

Figure 3 summarises the processing steps for estimating bathymetric information from SPOT-6 satellite imagery. The first two steps involve converting the DNs to reflectance values and correcting both atmospheric and sun glint errors. These steps are performed in an ENVI environment. The proposed approaches for estimating bathymetry are then conducted in a MATLAB environment. All the statistical analysis was also performed in a MATLAB environment.

The ratio method was applied using the ratio between corrected green and red band logarithms of the field points and were correlated to the water depth values at the same points yielding the following equation:

$$
Z=-86.21 *(1000(\ln \text { green })) /(1000(\ln \text { red }))+260.6
$$

This equation can be used to calculate bathymetry over 


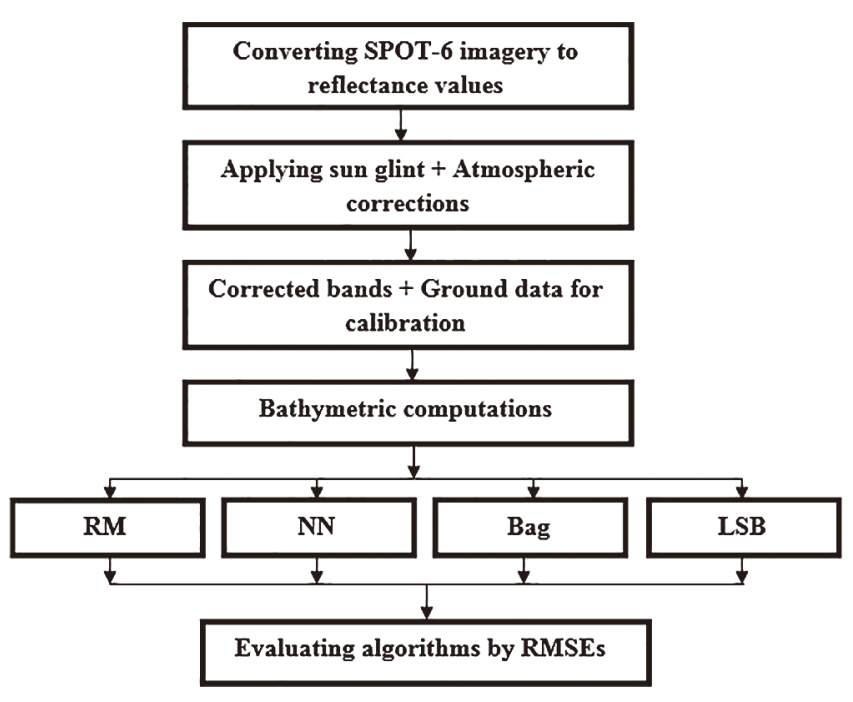

Figure 3. Workflow processing steps for detecting bathymetry from SPOT-6 satellite imagery

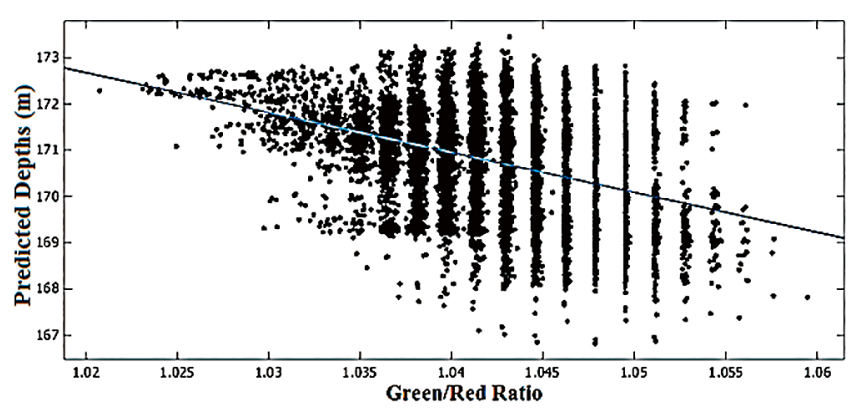

Figure 4. Ratio method continuous fitted model

the whole study area. The algorithm yielded $\mathrm{R}^{2}=0.144$, Bias $=0.011$, and $\mathrm{RMSE}=1.03 \mathrm{~m}$. The ratio model is presented in Figure 4.

The MLPBP supervised neural network with the LevenbergMarquard training algorithm was applied. Corrected green, red, blue/red, and green/red band logarithms were used as the input layer and water depths as the output layer. The data set was divided into random samples with $75 \%$ for training and $25 \%$ for testing. The log sigmoid function was used with the hidden layer, 10 neurons were selected after many trials to get the optimum number of neurons, and the linear function with the output layer. The NN results were $\mathrm{R}^{2}=0.215$, Bias $=0.002$, and RMSE $=0.97 \mathrm{~m}$. Results for the NN fitted model are shown in Figure 5.

An LSB ensemble was applied using the corrected green, red, blue/red, and green/red band logarithms as input values and water depths as output values. The data set was divided into separate random samples with $75 \%$ training and $25 \%$ testing sets. The most suitable number of regression trees was selected after several trials based on the least RMSE and most accurate $\mathrm{R}^{2}$ value. The best number of trees was 50 and resulted in $\mathrm{R}^{2}=0.204$, Bias $=-0.009$, and $\mathrm{RMSE}=0.99 \mathrm{~m}$, as shown in Figure 6.

A Bag ensemble was applied using the corrected green, red, blue/red, and green/red band logarithms as input values and water depths as output values. The data set was divided

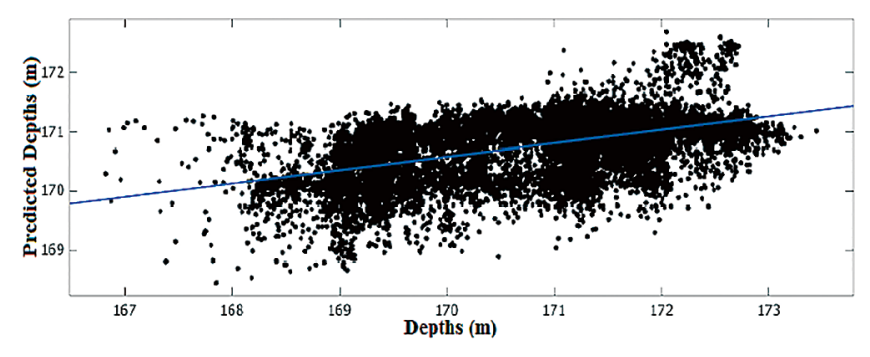

Figure 5. NN continuous fitted model

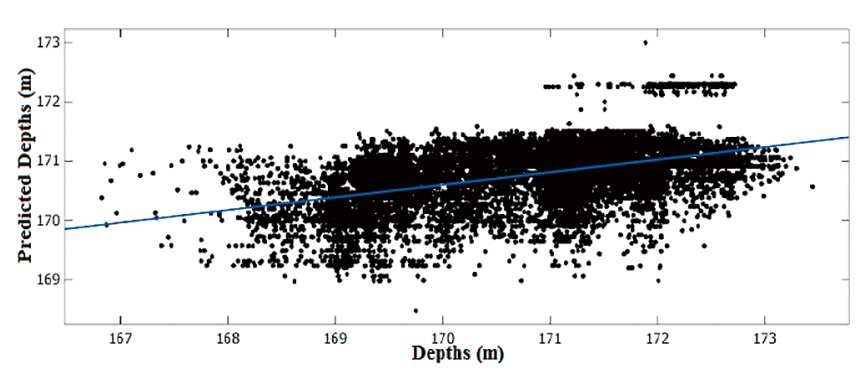

Figure 6. LSB ensemble continuous fitted model

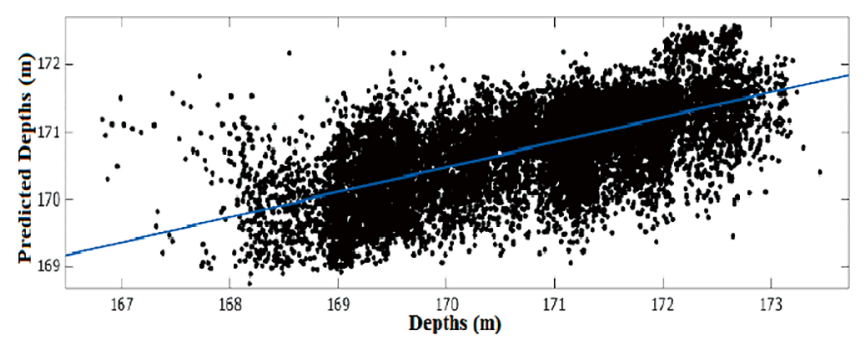

Figure 7. Bagging ensemble continuous fitted model

into separate random samples with $75 \%$ training and $25 \%$ testing sets. The most suitable number of regression trees was selected after several trials based on the least RMSE and most accurate $\mathrm{R}^{2}$ value. The optimum number of trees was 50 and yielded $\mathrm{R}^{2}=0.412$, Bias $=-0.004$ and $\mathrm{RMSE}=0.85 \mathrm{~m}$, as shown in Figure 7.

Figure 8 represents the results of all bathymetric approaches maps and true depths map.

Table I illustrates the RMSEs, $\mathrm{R}^{2}$, and bias of all proposed methods for bathymetry detection.

Selecting suitable bands for bathymetry was performed using a statistical analysis process to study the correlation between water depth and the four SPOT-6 imagery bands. The red and green band logarithms proved a strong correlation with water depth. This strong correlation has been argued for previously by many researchers (e.g. Doxani et al., 2012; Sánchez-Carnero et al., 2014).

In addition, the RM model uses the ratio between two bands, the ratio between green and red bands or the ratio between blue and red bands. Some studies (Stumpf et al., 2003; Su et al., 2008) argued for the validity of using these bands by the RM for detecting bathymetry. In our study the $\mathrm{RM}$ with green and red bands logarithms gave more accurate results than blue and green bands logarithms with RMSE of $1.03 \mathrm{~m}$. 


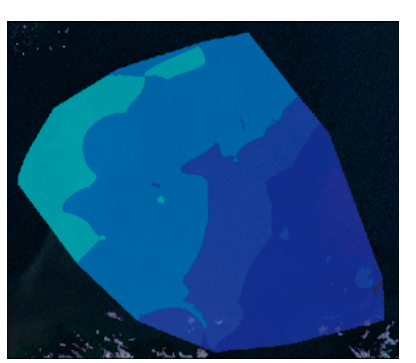

Elevations MSL

$\square 166.50 \mathrm{~m}$

$167.50 \mathrm{~m}$

$\square 170.50 \mathrm{~m}$

$171.50 \mathrm{~m}$

$172.50 \mathrm{~m}$

$173.50 \mathrm{~m}$

(a)

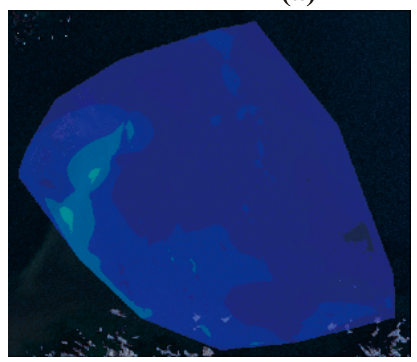

(b)

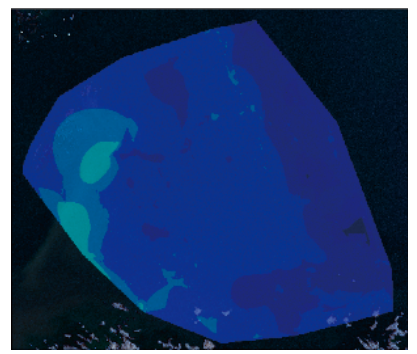

(d)

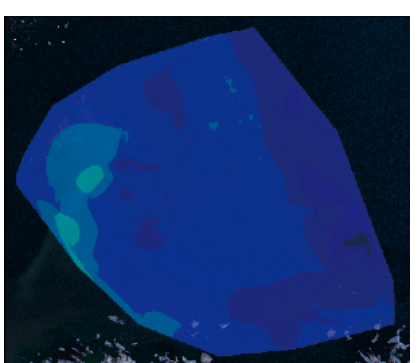

(c)

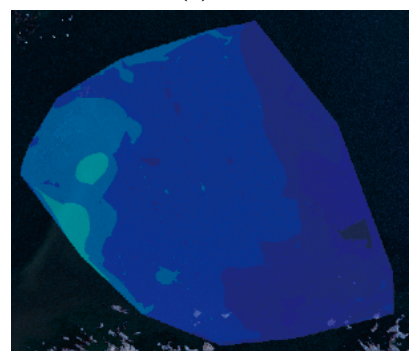

(e)
Figure 8. Results of all bathymetric approaches related to MSL (a) true depths (b) RM (c) NN (d) LSB (e) Bag

Table I. The RMSEs, $\mathrm{R}^{2}$, and bias of all proposed methods for bathymetry detection

\begin{tabular}{ccccc}
\hline Methodology & RM & NN & LSB & Bag \\
\hline RMSE (m) & 1.03 & 0.97 & 0.99 & 0.85 \\
R $^{2}$ & 0.144 & 0.215 & 0.204 & 0.412 \\
Bias & 0.011 & 0.002 & -0.009 & -0.004 \\
\hline
\end{tabular}

On the other hand, NN algorithms execute the correlation between the multilayer of the imagery bands as input and water depth as output. Many researchers (Mehdi et al., 2013; Ozçelik and Arısoy, 2010) have proposed a precedence of $\mathrm{NN}$ to RM and Lyzenga methods as the former exhibits the highest correlation between the imagery data bands and in situ water depth values. In these previous studies the visible bands were used as input layers without corrections or using any ratios. However, in this study, and after many trials, an integration between green, red bands and band ratios of blue /red and green/red as input layers was proposed. This integration gives the most accurate results using $\mathrm{NN}$ with a RMSE of $0.97 \mathrm{~m}$. The main drawback of NN is that many trials are needed to find the best weights for correlation as it is an unstable model with high fluctuations of RMSE and $\mathrm{R}^{2}$ from one trial to another.

The LSB and Bag are fitting ensembles of regression tree algorithms that have two different hypotheses for assembling regression trees. The LSB performs in a sequence by focusing on the missed regression values of the former trees. Conversely, the Bag fitting ensemble averages regression trees made from a bootstrapped random selection from input data. In these two approaches, the optimal number of regression trees was selected after successive trials with several numbers of trees $(10,20,30 \ldots 100)$, and the best values were reached with 50 trees. The randomness of the regression trees and the splitting of data into training and testing sets guarantee that the ensembles will not be overfitting the input data. As in $\mathrm{NN}$, the same input bands were selected after many trials based on the lowest RMSE and highest $\mathrm{R}^{2}$ giving $0.99 \mathrm{~m}$ and $0.85 \mathrm{~m}$ for LSB and Bag, respectively.

To compare our study with previous counterparts, several items must be considered, i.e., the depth range of the study area, water quality, bottom surface type, image geometric, radiometric resolution, and the availability of field points in the study area. For instance, Linda et al. (2011) applied a neuro-fuzzy algorithm to a QUICKBIRD image with $0.6 \mathrm{~m}$ spatial resolution over a sandy on-shore clear water area. The algorithm yielded standard deviation of $0.36 \mathrm{~m}$ at a depth range of $18 \mathrm{~m}$. Sánchez-Carnero et al. (2014) used a traditional linear approach to estimate depths over a sandy turbid area with a 6-m range. The RMSE was approximately $0.88 \mathrm{~m}$. In this study the clay bottom surface of the lake entrance zone and the high turbidity are considered the main factors which affected the detected depths and resulted in high RMSE values for all approaches.

Adding the ratios between bands to corrected bands as input for all algorithms which have more than two bands as input improves the results considerably. The three approaches of NN, LSB, and Bag are more accurate than the conventional Ratio method. In addition, the Bag algorithm outperforms all methods and is more stable.

Further research could consider applying the same concepts described here to clearer water areas to achieve more accurate RMSE results. Although these RMSE results are relatively high, they could be used for lake management and navigation purposes especially in the entrance zone. This area presents many difficulties for mapping field bathymetry because of its shallowness, its continuous sediment movements and the synoptic coverage of satellite imageries.

\section{CONCLUSIONS}

In this study, new methods for detecting bathymetry are proposed and compared with conventional approaches. These algorithms are known as Bag and LSB, and use corrected red, green, green/red, and blue/red bands from atmospheric and sun glint errors as input values. The algorithms were validated using SPOT-6 imagery over the entrance zone of Lake Nubia. The study area was extremely turbid and had a clay bottom surface and a depth range of $6.5 \mathrm{~m}$. The new approaches were compared with two conventional approaches: the NN and RM. The Bag algorithm yielded RMSE of $0.85 \mathrm{~m}$ whereas the LSB, NN, and RM results were $0.99,0.97$, and $1.03 \mathrm{~m}$, respectively. These results proved that the proposed approaches outperformed the conventional RM method. In addition, results improved when using the ratios between bands with the corrected bands as 
input for all approaches. Finally, the Bag algorithm proved to perform uniquely well in comparison to all tested methods, including the NN approach.

\section{ACKNOWLEDGMENTS}

The first author would like to thank the Egypt-Japan University of Science and Technology (E-JUST) and JICA for their support and for offering the tools needed for this research. This work was supported by JSPS "Core-to-Core Program, B. Asia-Africa Science Platforms".

\section{REFERENCES}

Ananth R. 2004. The Levenberg-Marquardt Algorithm.8. http:// www.ananth.in/Notes_files/lmtut.pdf. Last access October 20, 2015

Behzad S. 2014. Predicting the trend of land use changes using artificial neural network and Markov chain model (Case Study: Kermanshah City). Research Journal of Environmental and Earth Sciences 6: 215-226.

Breiman L. 1996. Bagging predictors. Machine Learning 24: 123140. DOI: 10.1023/A:1018054314350.

Doxani G, Papadopouloua M, Lafazania P, Pikridasb C, Tsakiri S. 2012. Shallow-water bathymetry over variable bottom types using multispectral Worldview-2 image. Proceeding of XXII ISPRS Congress, 2012 Melbourne, Australia; XXXIX-B8.

Hastie T, Tibshirani R, Friedman J. 2008. The Elements of Statistical Learning Data Mining, Inference, and Prediction. 2nd ed. Springer-Verlag, Stanford, California, USA; 337-388.

Hedley J, Harborne A, Mumby P. 2005. Simple and robust removal of sun glint for mapping shallow-water benthos. International Journal of Remote Sensing 26: 2107-2112. DOI: 10.1080/ 01431160500034086.

Ihler A. 2012. Ensembles of learners. Presented at Machine Learning and Data Mining, Bern ICS Information and Computer Science, 2012 University of California, USA. https://www.coursehero.com/file/7352307/10-ensembles/. Last access November 15, 2015.

Inoue A, Kilian L. 2005. How useful is bagging in forecasting economic time series? A case study of US CPI inflation. CEPR Discussion Paper No. 5304. http://ssrn.com/abstract=872856. Last access October 14, 2015.

Leu L, Chang H. 2005. Remotely sensing in detecting the water depths and bed load of shallow waters and their changes. Ocean Engineering 32: 1174-1198. DOI: 10.1016/j.oceaneng. 2004.12.005.

Linda C, Andrea M, Marco C. 2011. Approaching bathymetry estimation from high resolution multispectral satellite images using a neuro-fuzzy technique. Journal of Applied Remote Sensing 5: 0535151-05351515. DOI: 10.1117/1.3569125.

Lyzenga D. 1978. Passive remote sensing techniques for mapping water depth and bottom features. Applied Optics 17: 379383. DOI: 10.1364/AO.17.000379.

Lyzenga D. 1981. Remote sensing of bottom reflectance and other attenuation parameters in shallow water using aircraft and Landsat data. International Journal of Remote Sensing 2:
71-82. DOI: 10.1080/01431168108948342.

Lyzenga D. 1985. Shallow-water bathymetry using combined Lidar and passive multispectral scanner data. International Journal of Remote Sensing 6: 115-125. DOI: 10.1080/ 01431168508948428.

McIntyre L, Naar F, Carder L, Donahue T, Mallinson J. 2006. Coastal bathymetry from hyperspectral remote sensing data: Comparisons with high resolution multibeam bathymetry. Marine Geophysical Researches 27: 129-136. DOI: 10.1007/ s11001-005-0266-y.

Mehdi G, Tiit K, Abbas E, Ali A, Babak N. 2013. Remotely sensed empirical modeling of bathymetry in the southeastern Caspian Sea. Remote Sensing 5: 2746-2762. DOI: 10.3390/rs5062746.

Michael J. 2009. Depth Derivation from the Worldview-2 Satellite Using Hyperspectral Imagery. Master's Thesis, California, Naval Postgraduate School; 69.

Mochizuki S, Murakami T. 2012. Accuracy comparison of land cover mapping using the object oriented image classification with machine learning algorithms. 33rd Asian Conference on Remote Sensing. Ambassador City Jomtien Hotel, Pattaya, Thailand.

Ozçelik C, Arısoy Y. 2010. Remote sensing of water depths in shallow waters via artificial neural networks. Estuarine, Coastal and Shelf Science 89: 89-96. DOI: 10.1016/j.ecss.2010.05. 015 .

Ozlem A, Oguz G. 2012. Classification of multispectral images using Random Forest algorithm. Journal of Geodesy and Geoformation 1: 105-112. DOI: 10.9733/jgg.241212.1.

Quinlan J. 2006. Bagging Boosting and C4.5. http://www.cs. utah.edu/ piyush/teaching/Quinlan.AAAI96.pdf. Last access September 8, 2015.

Rumelhart D, Hinton G, Williams R. 1986. Learning internal representations by error propagation. In Advances in Parallel Distributed Processing, Rumelhart D, Hinton G, Williams R (eds). MIT Press Cambridge: MA, USA; 318-362.

Sánchez-Carnero N, Ojeda-Zujar J, Rodríguez-Pérez D, MarquezPerez J. 2014. Assessment of different models for bathymetry calculation using SPOT multispectral images in a highturbidity area: the mouth of the Guadiana Estuary. International Journal of Remote Sensing 35: 493-514. DOI: 10.1080/01431161.2013.871402.

Sheela A, Letha J, Sabu J, Jairaj P, Justus J. 2013. Lake bathymetry from Indian remote sensing (P6-LISS III) satellite imagery using artificial neural network model. Lakes \& Reservoirs: Research and Management 18: 145-153. DOI: 10.1111/lre. 12027.

Shivali K, Vishakha K. 2014. Classification of multispectral satellite images using ensemble techniques of bagging, boosting and ada-boost. Circuits, Systems, International Conference on Communication and IT Applications (CSCITA), 253-258. DOI: 10.1109/CSCITA.2014.6839268.

Stumpf R, Holderied K, Sinclair M. 2003. Determination of water depth with high-resolution satellite imagery over variable bottom types. Limnology and Oceanography 48: 547-556. DOI: 10.4319/1o.2003.48.1_part_2.0547.

Su H, Liu H, Heyman W. 2008. Automated derivation for bathymetric information for multispectral satellite imagery using a non-linear inversion model. Marine Geodesy 31: 281-298. DOI: $10.1080 / 01490410802466652$. 